\title{
Computerized Histologic Image Based Risk Predictor (CHIRP): Identifying Disease Aggressiveness Using Sub-visual Image Cues from Image Data
}

\author{
Anant Madabhushi
}

Center for Computational Imaging and Personalized Diagnostics, Department of Biomedical Engineering, Case Western Reserve University. Cleveland, OH, USA

With the advent of digital pathology, there is an opportunity to develop computerized image analysis methods to not just detect and diagnose disease from histopathology tissue sections, but to also attempt to predict risk of recurrence, predict disease aggressiveness and long term survival. At the Center for Computational Imaging and Personalized Diagnostics, our team has been developing a suite of image processing and computer vision tools, specifically designed to predict disease progression and response to therapy via the extraction and analysis of image-based "histological biomarkers" derived from digitized tissue biopsy specimens. These tools would serve as an attractive alternative to molecular based assays, which attempt to perform the same predictions. The fundamental hypotheses underlying our work are that: 1) the genomic expressions detected by molecular assays manifest as unique phenotypic alterations (i.e. histological biomarkers) visible in the tissue; 2) these histological biomarkers contain information necessary to predict disease progression and response to therapy; and 3) sophisticated computer vision algorithms are integral to the successful identification and extraction of these biomarkers. Brief description of recent findings for a few different tumors are summarized below.

1. Predicting outcome in p16+ Oropharyngeal tumors $(\mathbf{N}=\mathbf{1 6 0})$ : We have previously leveraged the relationship between disease aggressiveness and tumor morphology by developing novel graph-based techniques to characterize the 2D arrangement of nuclei in breast and prostate cancers. More recently, we have developed sophisticated cell cluster graphs (CCGs) that quantify localized tissue architecture [1] and have been shown to be more flexible and robust than traditional graphs for differentiating disease aggressiveness and patient outcome in prostate and oropharyngeal cancer histopathology [1, 2]. In addition, we have shown that a spatially-aware CCG model that constructs graphs separately for epithelial and stromal compartments may yield prognostic information in oropharyngeal cancers [3] (Figure 1). With CCGs, we leverage advanced graph metrics (e.g., Hosoya index), sub-visual features that attempt to quantify higher-order characteristics (e.g., graph cyclicity, intersection) of tissue structure and tumor morphology not visually discernible by a pathologist. Our team has shown that a computerized histologic image-based predictor could distinguish $(\mathrm{AUC}=0.89$ ) between progressors and nonprogressors from among a cohort of $\mathrm{N}=160$ oropharyngeal squamous cell carcinoma patients using spatial architecture features of nuclear arrangement [2].

2. Predicting biochemical recurrence based off Ibris analysis of radical prostatectomy whole slides (N=40): For a cohort of $(\mathrm{N}=40)$ grade, stage matched tumors, the Madabhushi team showed that gland tensors were able to accurately predict 5 year biochemical recurrence over $75 \%$ of the time. The nuclear and gland tensors both outperform the Kattan nomogram in terms of predicting PSA recurrence (see Figures 4(d), (h)). In this PLOS One paper [4], Madabhushi et al showed that glands are more chaotically arranged in aggressive versus indolent intermediate risk prostate cancer. The entropy associated with these "gland angularity features" was hence higher in aggressive and lower in indolent disease (see Figures 2(e), (f)). 


\section{Predicting biochemical recurrence based off image analysis of radical prostatectomy TMAs} (N=80): Figures 2(d), (h) shows Kaplan Meier curves, generated by the Madabhushi group, for the prediction of 5 year PSA recurrence following surgery [1] in $(n=80)$ prostate cancer patients based off nuclear tensor features from tissue microarrays.

\section{References:}

[1] Ali, S., et al, Cell cluster graph for prediction of biochemical recurrence in prostate cancer patients from tissue microarrays. 2013.

[2] Lewis, J.S., Jr., et al, Am J Surg Pathol, 2014. 38(1): p. 128-37.

[3] Ali, S., J. Lewis, and A. Madabhushi, Spatially Aware Cell Cluster(SpACCl) Graphs: Predicting Outcome in Oropharyngeal p16+ Tumors, in Medical Image Computing and ComputerAssisted Intervention - MICCAI 2013, K. Mori, et al., Editors. 2013, Springer Berlin Heidelberg. p. 412-419.

[4] Lee G, et al, PLoS One. 2014 May 29;9(5):e97954. doi: 10.1371/journal.pone.0097954. eCollection 2014.

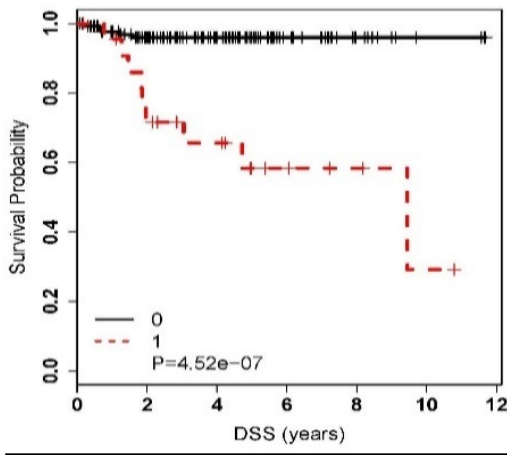

Figure 1: Published results show KM curves for prediction of progression in $\mathbf{N}=\mathbf{1 6 0}$ head \& neck cancers. MsCHIP on H\&E outperform TNM-stage prediction.

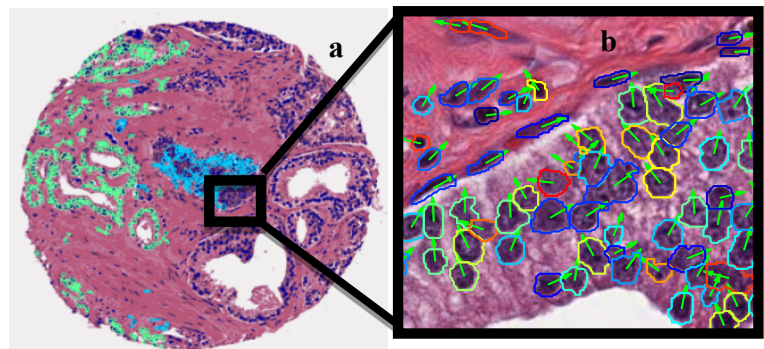

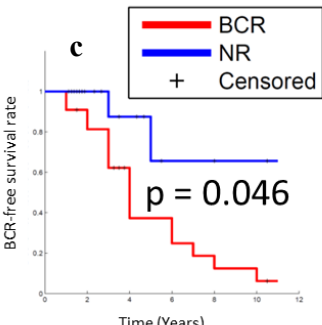

Time (Years)
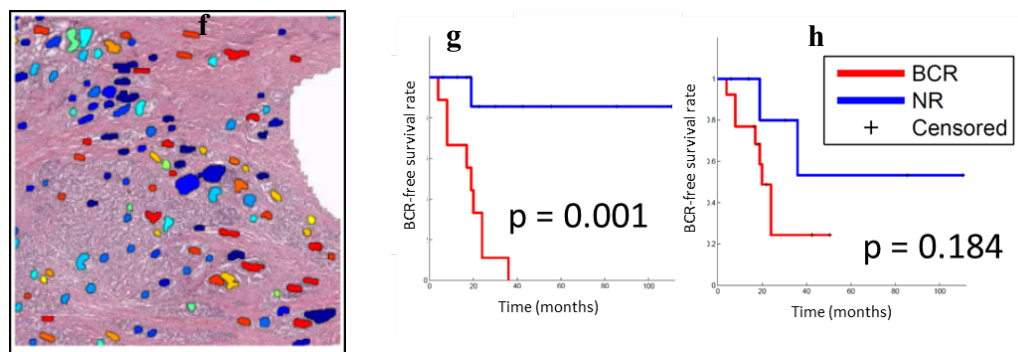

Figure 2: Quantitative histomorphometric features from standard H\&E tumor images can predict disease behavior and outcome (from Ibris team). (a) Cell cluster graph showing connectivity between individual nuclei, (b) nuclear tensors showing orientation of individual cells which the Ibris team have shown to be more chaotically arranged in aggressive versus indolent disease. Kaplan Meier curves (c) for nuclear tensor feature in separating $(\mathbf{N}=\mathbf{8 0})$ prostate cancer patients with and without 5 year PSA recurrence is significantly different and further improves by (d) including tumor stage. (e) Gland orientations in prostate pathology were shown by the Madabhushi group to be more chaotically arranged in more aggressive vs indolent disease. (f) Entropy of gland orientations in aggressive disease is hence higher (see different colors) and allows for $(\mathrm{g})$ significant separation between $(\mathbf{N}=\mathbf{4 0})$ intermediate risk cancers with, without (NR) 5 year BCR, compared to (h) Kattan nomogram. 